\title{
Flow Estimation Using Subharmonics of Microbubbles
}

\author{
Mentzung Lo, Dung-Han Tsai, and Jenho Tsao. \\ Graduate Institute of Communication Engineering, National Taiwan University Taipei, \\ Taiwan, R.O.C.
}

Abstract - It has been observed that the subharmonic signal of the microbubbles has a significant contrast-to-tissue ratio. The flow velocity can be evaluated precisely even without wall filter for subharmonics since the vessels containing the microbubbles would be distinguishable from the surrounding tissues. However, the microbubble cannot generate the subharmonics unless the applied pressure exceeds the required onset threshold. Moreover, it will undergo cavitation if the acoustic pressure is over the cavitation threshold. While the microbubble moves through the sample volume, there are three different regions inside the excitation beam. The beam-weighted pressure is below the onset threshold, between the onset and cavitation thresholds, and over the cavitation threshold. Since the subharmonics can occur only when the beam-weighted pressure is between the onset and cavitation thresholds, the observation time for subharmonics is shorter than that for the fundamental. Our numerical results showed that the onset threshold is very close to the cavitation threshold. Moreover, some microbubbles are found to generate subharmonics and undergo cavitations simultaneously. Our mathematical analysis showed that the consecutive received signals would have very low correlation for the subharmonics. This would broaden the Doppler power spectrum bandwidth of the subharmonics. The experimental data

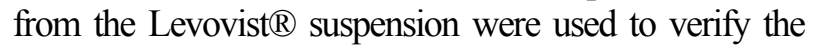
theoretical predictions. It can be shown that the absolute value of the normalized autocorrelation (first lag) of Doppler signals for the subharmonics was much smaller than the fundamental and the second harmonics when the emitted frequency is $2.1 \mathrm{MHz}$ and the acoustic pressure is $0.8 \mathrm{Mpa}$. In addition, it is found that the correlation value is always below 0.3 when the emitted frequency is swept from 1.5 to $2.5 \mathrm{MHz}$ and the applied pressure is varied from 0.1 to $1.6 \mathrm{Mpa}$.

\section{INTRODUCTION}

Since the contrast of microbubbles to the surrounding tissues can be enhanced with harmonics, the slow velocity can be evaluated accurately even without the precise wall filter. Therefore, some researches were devoted to the applications on flow velocity estimation with harmonics
[1]. Unlike the second harmonics components, the subharmonics cannot be excited from the tissues [2]. Therefore, the vessels containing the microbubbles will be more easily distinguished from the surrounding tissues and then the flow velocity in the vessels will be assessed more precisely, if the subharmonics rather than the second harmonics are used for velocity estimation. Based on the previous theoretical studies, both the microbubbles and encapsulated agents are difficult to generate the subharmonics unless the acoustic pressure exceeds the onset threshold. Moreover, while the acoustic pressure exceeds the cavitation threshold, the microbubble would grow explosively and then collapse [3].

Previous researches have awarded that there is a close relationship between the two thresholds. However the influence of the two thresholds in flow estimation has not been specified yet. It is shown in this study that the two thresholds would reduce the transit time for the microbubbles, which can produce the subharmonics, resulting in the broadening of the Doppler power spectrum. In this paper, the reduced observation time effect on autocorrelator used for velocity estimation is demonstrated.

\section{OBSERVATION TIME ANALYSIS}

\section{Correlation property of harmonics}

Assuming that the emitted pulse length is sufficiently long, the continuous form of Doppler signal obtained from harmonic components in RF domain at $t_{s}$ can be expressed as

$$
\begin{aligned}
& u_{x}(t)=\sum_{k} \sigma_{k, f_{c} \cdot 2^{\Gamma}}{ }^{1 / 2} \cdot e^{j\left(2 \pi \times f_{d_{k}} t+\phi_{k} \cdot 2^{\Gamma}\right)} B_{k}\left(t-\xi_{k}\right) \\
& B_{k}\left(t-\xi_{k}\right)=T\left[\left(t-\xi_{k}\right), f_{c}\right] \cdot T\left[\left(t-\xi_{k}\right),\left(f_{c} \cdot 2^{\Gamma}\right)\right] \\
& f_{d_{k}}=\frac{2 v_{k}}{C} f_{c} \cdot 2^{\Gamma}, \phi_{k}=2 \pi f_{c}\left(t_{s}-\varsigma_{k}\right)
\end{aligned}
$$

Where $f_{c}$ and $\mathrm{A}$ are the center frequency and amplitude of the transmission, respectively, $\Gamma=-1,0,1$ for the subharmonics, the fundamental and second harmonics, respectively, $\sigma_{s c,\left(k, f_{c} \cdot 2^{\mathrm{r}}\right)}$ is the backscattering cross-section of the $\mathrm{k}$-th microbubble for the corresponding harmonic frequencies, $\varsigma_{k}$ is the time it 
takes sound to propagate between the k-th microbubble and the probe, $\xi_{k}$ is the time it takes the $\mathrm{k}$-th microbubble to arrive the beam axis, $T(\bullet)$ is the transmitted beam weighting of the transducer; $\mathrm{C}$ and $T_{s}$ are sound velocity and the pulse repetition period, respectively. The autocorrelation function of Doppler signal in (1) is given by

$$
\begin{aligned}
& R_{x}(\tau)=E\left[u_{x}(t) u_{x}{ }^{*}(t-\tau)\right] \\
& \approx \sum_{l=k} E\left[\sigma_{k, f_{c} \cdot 2^{\Gamma}} \cdot B_{k}\left(t-\xi_{k}\right) B_{k}{ }^{*}\left(t-\xi_{k}-\tau\right)\right] e^{j 2 \pi f_{d_{k}} \tau}
\end{aligned}
$$

The above equation holds under the assumption that $\phi_{k}$ is uniformly distributed over $[-2 \pi, 2 \pi]$. For the simplification of derivation, we assume that $E\left[B\left(t-\xi_{k}\right) B^{*}\left(t-\xi_{k}-\tau\right)\right]=e^{-\tau \cdot v_{k} / L}$ under small $\tau$, where $L$ is the effective length of range cell. Assuming that $E\left[\sigma_{k, f_{c} \cdot 2^{\Gamma}}\right]=E\left[\sigma_{f_{c} \cdot 2^{\Gamma}}\right]$, each velocity component has the same weighting $(w), f_{d_{k}}=2\left(v_{d}+\Delta v \cdot k\right) \cdot f_{c} \cdot 2^{\Gamma} / C$, and

$$
\begin{aligned}
\Delta \theta=2 & \pi \frac{2 \Delta v}{C} f_{c} 2^{\Gamma} \tau, \text { we get } \\
R_{x}(\tau) & =w \cdot E\left[\sigma_{f_{c}, 2^{\Gamma}}\right] e^{j 2 \pi \frac{2 v_{d}}{C} f_{0} \tau} e^{-\frac{\tau \cdot v_{d}}{L}} \sum_{k=-N}^{N} e^{\frac{\tau \Delta v \cdot k}{L}} e^{j 2 \pi \frac{2 \Delta \cdot \cdot k}{c} f_{0} \tau} \\
& =w \cdot E\left[\sigma_{f_{c} 2^{\Gamma}}{ }^{2}\right] e^{j 2 \pi \frac{2 v_{d}}{c} f_{0} \tau} e^{\frac{-\tau \cdot v_{d}}{L}}\left(1-e^{N \Phi}\right) /\left(1-e^{\Phi}\right) \\
\Phi & =(-\alpha+j) \Delta \theta, \quad \alpha=\lambda / 4 \pi L, \quad \lambda=C / f_{c} 2^{\Gamma}
\end{aligned}
$$

The standard deviation of Doppler power spectrum density used to represent the Doppler bandwidth is proportional to $\left(1-\left|R_{x}\left(T_{s}\right) / R_{x}(0)\right|\right)^{0.5}$, which can be approximated as

$$
\begin{aligned}
& \sqrt{1-\left|\frac{R_{x}\left(T_{s}\right)}{R_{x}(0)}\right|} \approx \sqrt{1-e^{\frac{-T_{s} v_{d}}{L}} \sin c\left(\left.N \cdot \Delta \theta\right|_{\tau=T_{s}}\right)} \\
& \approx \sqrt{\left\{1-\left[1-\left(\left.N \Delta \theta\right|_{\tau=T_{s}}\right)^{2} / 3 !+\ldots\right]\right\}},
\end{aligned}
$$

when $L$ is sufficiently wide (i.e. $\alpha<<1$ ). If the higher order terms are neglected, $\left(1-\left|R_{x}\left(T_{s}\right) / R_{x}(0)\right|\right)^{0.5}$ will be proportional to the velocity spread (i.e. $\mathrm{N}$ ) and harmonic frequency (i.e. $f_{c} \cdot 2^{\Gamma}$ ). In other words, when the length of range cell is sufficiently long relative to the moving distance for a microbubble during the two consecutive firings (i.e. $L \gg>T_{s} v_{d}$ ), the ratio of $\left(1-\left|R_{x}\left(T_{s}\right) / R_{x}(0)\right|\right)^{0.5}$ for the subharmonics to that for the fundamental approaches to 0.5 .

According to (3), if the length of range cell is so short that the real part of $\Phi$ cannot be neglected, the transit time effect will dominate the autocorrelation function. Consequently, (4) cannot hold and $\left(1-\left|R_{x}\left(T_{s}\right) / R_{x}(0)\right|\right)^{0.5}$ is no longer proportional to the harmonic frequency and velocity spread, moreover, which reduces the value of $\left|R_{x}\left(T_{s}\right) / R_{x}(0)\right|$ and in consequence the estimated flow velocity is unreliable.

\section{Numerical results of onset and cavitation thresholds}

The theoretical instantaneous radius of RPNNP is evaluated numerically using MATLB ordinary difference equation solver, and Table I lists the parameters for the numerical evaluation. While the driving frequency is fixed at $2.5 \mathrm{MHz}$, Fig. 1 shows that the subharmonic backscatter normalized to the fundamental one as a function of the applied pressure. It can be observed that the normalized subharmonic intensity undergoes rapid growths as the applied pressure exceeds certain threshold. According to the intensity of the subharmonics (normalized to the fundamental) for different applied pressure, a suitable onset threshold is selected at $-40 \mathrm{~dB}$. The corresponding onset threshold for bubbles with different radius is given as dashed line in Fig. 2.

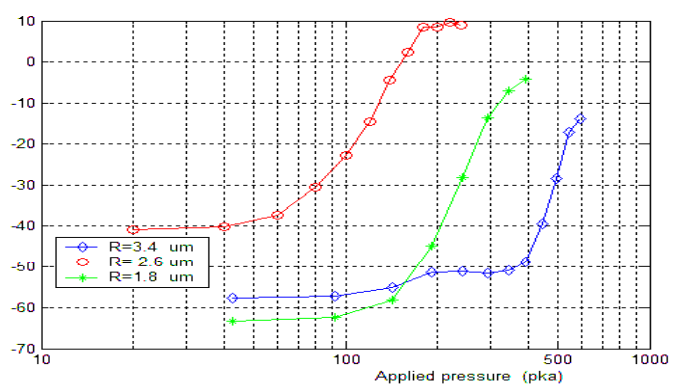

Fig.1. The normalized intensity of the subharmonics $(\mathrm{dB})$

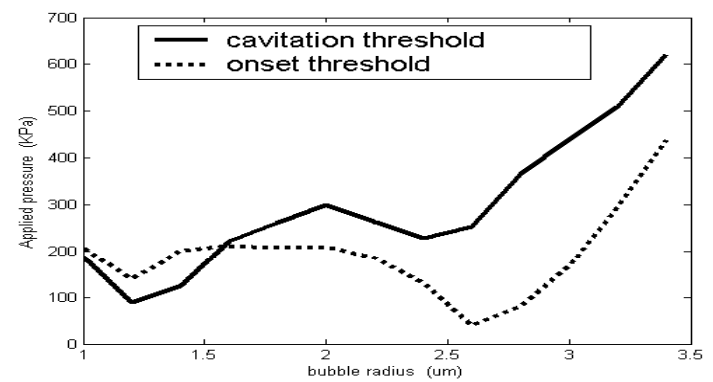

Fig 2. The onset threshold (dashed line) and transient collapse threshold (solid line) against initial bubble radius.

If the pressure is high enough, the pressure in the liquid will become negative. Consequently, the bubble will expand to the maximum size $\left(R_{\max }\right)$ and undergo collapse. To achieve this, $R_{\max } / R_{0}$ was suggested to exceed 2 [4]. According to the numerical results, we can obtain the maximum radius as a function of applied pressure for bubbles with different initial radius, and then Fig. 2 illustrates the pressure threshold of transient collapse as a function of bubble initial radius with solid line, using the 
criteria that $R_{\max } / R_{0}$ must exceed 2. It is observed that the threshold increases with bubble radius except for two radii corresponding to the cases when the resonant frequencies are around the driving frequency and half the driving frequency. The inspection of the behavior of onset threshold shows that the number of bubbles, which can be excited significant subharmonic oscillations, increases with the acoustic pressure. However, under high pressure, most bubbles, which can be excited subharmonics, would undergo cavitation before they reach the beam axis (maximum beam intensity). Moreover, according to Fig.2, the difference between the onset threshold and cavitation threshold for most of the bubbles, which can be excited to have substantial subharmonics, is very small. Therefore, according to (3), the value of $\left|R_{x}\left(T_{s}\right) / R_{x}(0)\right|$ for the subharmonics is reduced excessively and the Doppler bandwidth for the subharmonics broadens apparently. Consequently, the term $\left(1-\left|R_{x}\left(T_{s}\right) / R_{x}(0)\right|\right)^{0.5}$ for the subharmonics may not be able to indicate the velocity spread.

\section{EXPERIMENTAL STUDIES}

\section{Material and methods}

The block diagram of the experimental procedure is shown in Fig. 3. All measurements were carried out with the injection of microbubbles into the circulating system. Two types of microbubbles, including free gas and Levovist ${ }^{\mathbb{B}}$ were conducted.

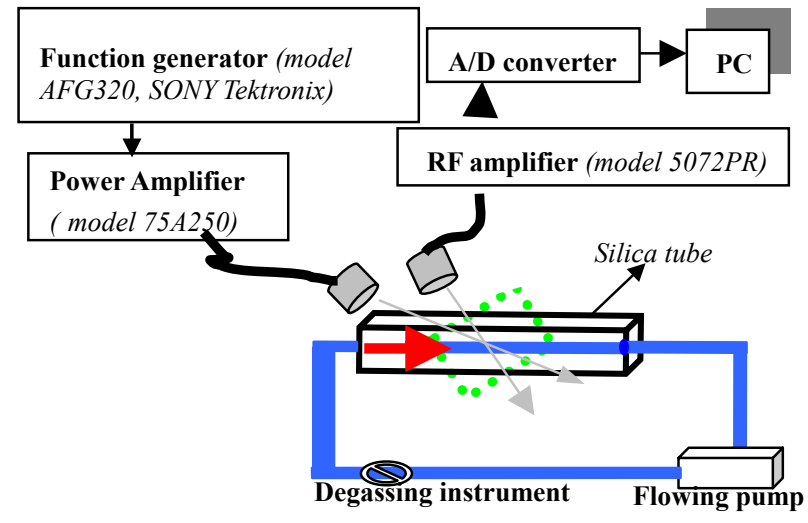

Fig.3 The diagarm of the experimental set-up

\section{The excessive decorrelation for the subharmonic}

In this experiment, the center frequency of the transmitted signals is $1.9 \mathrm{MHz}$. The Gaussian bursts with the standard deviation of $10 \mu \mathrm{s}$ and the peak pressure of $800 \mathrm{Kpa}$ are transmitted at a PRF of $5 \mathrm{KHz}$. The averaged power spectrum over 500 independent realizations from air bubbles was presented in Fig. 4(a). It is founded that the intensity of the subharmonics is greater than that of the second harmonics in our experimental condition (i.e. the subharmonics have higher SNR than the second harmonics). Fig.4 (b) compares the absolute values of autocorrelation function for Doppler data between the fundamental and subharmonics and second harmonics. Note that the Doppler data used to evaluate autocorrelation function has length of 480 samples, and the mean value and standard deviation of each component in the plot were calculated from three measurements. When the air bubbles in tube was replaced with the suspension of Levovist ${ }^{\circledR}$, the results of Levovist ${ }^{\mathbb{B}}$ are illustrated in the lower panels of Fig. 4. Note that the acoustic pressure is $800 \mathrm{KPa}$, and the center frequency is $2.1 \mathrm{MHz}$.

The calculated ratios of standard deviation of Doppler power spectrum density for the second harmonics to that for the fundamental are 1.8 and 1.9 for air bubbles and Levovist $^{\circledR}$, respectively. The ratios are close to 2 , which is the theoretical calculation under that velocity spread dominates the autocorrelation function of Doppler signals. However, as the theoretical suspension under that the transit time rather than velocity spread dominated the autocorrelation function of Doppler signals, the value of $|R(1) / R(0)|$ illustrated in Fig. 4 for the subharmonics is reduced substantially and even much smaller than that for the second harmonics

\section{Frequency and pressure-independence of the excessive decorrelation}

The acoustic pressure is fixed as $800 \mathrm{Kpa}$. We varied the emitted frequency from $1.5 \mathrm{MHz}$ to $2.1 \mathrm{MHz}$ with a spacing of $0.2 \mathrm{MHz}$. The values of $|R(1) / R(0)|$ for the fundamental, subharmonic and second harmonic components under different emitted frequencies were listed in Table II. Each component listed in Table II was obtained from seven separated measurements. Next, the insonified acoustic pressure was increased form 100 to $1800 \mathrm{Kpa}$ for $2.1 \mathrm{MHz}$ and $1.9 \mathrm{MHz}$. The mean values and standard deviations of $|R(1) / R(0)|$ for the subharmonics as a function of applied acoustic pressure under the insonified frequencies of $1.9 \mathrm{MHz}$ and 2.1 $\mathrm{MHz}$ are illustrated in Fig.5. Assuming that the velocity spread is fixed, the increased insonified frequency should broaden the bandwidth of Doppler signals theoretically. Therefore, as listed in Table II, the increment of insonified frequency reduces the value of $|R(1) / R(0)|$ for the fundamental and the second harmonics. However, the value of $|R(1) / R(0)|$ for the subharmonics is independent of emitted frequency. Moreover, the values of $|R(1) / R(0)|$ for the subharmonics under the insonified frequencies ranging from $1.5 \mathrm{MHz}$ to $2.1 \mathrm{MHz}$ are very small and not over 0.3 . 
Shi et al. indicated that the subharmonic component undergoes three stages: initiation, growth and saturation [5]. Such behavior of the subharmonics was presented in Fig. 5, which illustrated the intensity of subharmonic component against the applied acoustic pressure. The mean and standard deviation of each component in Fig. 5 was obtained from twelve separated measurements.
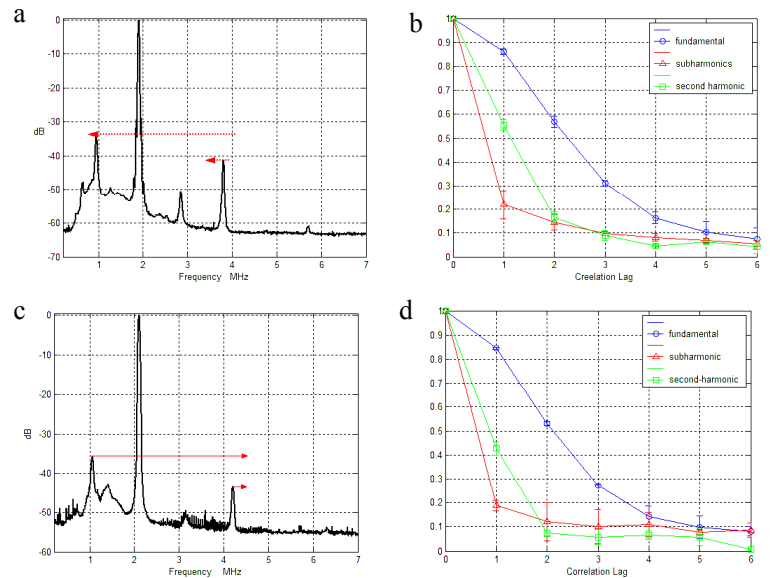

Fig.4. The averaged RF power spectrum for (a) free gas and (c) the suspension of Levovist ${ }^{\mathbb{R}}$, respectively. The autocorrelation function of Doppler data for (b) free gas and (d) the suspension of Levovist ${ }^{\circledR}$.
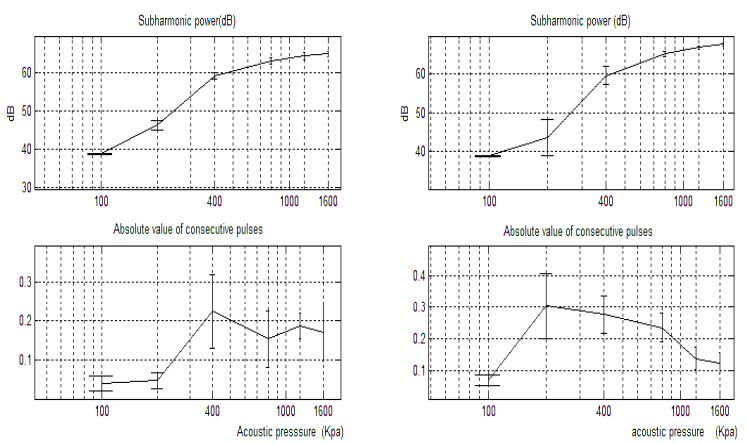

Fig.5. The backscatter of harmonics and normalized $|R(1)|$ for the suspension of Levovist ${ }^{\circledR}$ against the insonified pressure for (left) 2.1 $\mathrm{MHz}$ and for (right) $1.9 \mathrm{MHz}$.

We can observe that the subharmonics are insignificant in initiation, and then grow rapidly with applied pressure. Moreover, when the applied pressure exceeds a certain value, the subharmonics become saturated. The small vale of $|R(1) / R(0)|$ at initiation stage can be accused of the poor SNR of the subharmonics. However, there is only small increment in the value of $|R(1) / R(0)|$ while the subharmonic component is increased significantly. The value of $|R(1) / R(0)|$ has the maximum at growth stage, though the intensity of the subharmonics at growth stage is smaller than that at saturation stage.

\section{CONCLUSION}

The theoretical results have shown that the average transit time for the bubbles, which can be excited significant subharmonics, are reduced excessively. Moreover, experimental results show that the absolute value of normalized autocorrelation function of Doppler signals at unity lag for the subharmonics is much smaller than that for the fundamental and the second harmonics. The experimental results also show that the excessive decorrelation of Doppler signals for the subharmonics doesn't only belong to critical condition. Therefore, it is concluded that, compared to other harmonics, the average transit time of the bubbles for the subharmonics is too short to make the velocity estimation reliable. Moreover, velocity spread has less influence on the bandwidth of Doppler power spectrum of the subharmonics.

TABLE I. VALUES OF PARAMETERS FOR NUMERICAL EVALUATION OF RPNNP.

\begin{tabular}{ccccc}
\hline $\begin{array}{c}\text { Density of } \\
\text { liquid } \rho \text { Viscosity } \\
\text { of liquid } \mu\end{array}$ & $\begin{array}{c}\text { Surface } \\
\text { tension } \sigma\end{array}$ & $\begin{array}{c}\text { Polytroptic } \\
\text { exponent }\end{array}$ & $\begin{array}{l}\text { Ambient } \\
\text { pressure } p_{0}\end{array}$ \\
\hline $998 \mathrm{~kg} / \mathrm{m}^{3}$ & $0.001 \mathrm{~Pa} . \mathrm{s}$ & $0.068 \mathrm{~N} / \mathrm{m}$ & 1.07 & $101 \mathrm{KPa}$ \\
\hline
\end{tabular}

TABLE II. $|R(1) / R(0)|$ FOR AIR BUBBLES

\begin{tabular}{|l|rccc|}
\hline \multirow{2}{*}{} & \multicolumn{4}{|c|}{ Insonified frequency (MHz) } \\
\cline { 2 - 5 } & 1.5 & 1.7 & 1.9 & 2.1 \\
Fundamental & 0.924 & 0.896 & 0.865 & 0.818 \\
Second & 0.704 & 0.636 & 0.555 & 0.449 \\
Sub & 0.270 & 0.165 & 0.296 & 0.181 \\
\hline
\end{tabular}

\section{Reference}

[1] Pi Hsien Chang, K. K. Shung, S. Wu., and H. B. Levene, "Second Harmonic Imaging and Harmonic Doppler Measurements with Albunex," IEEE Trans, Ultrason., Ferroelect., Freq. Contr., Vol. 42, No. 6, pp. 1020-1027, 1995.

[2] P.M. Shankar, P.A. Krishna and V.L. Newhouse," Advantages of Subharmonic over second Harmonic Backcatter for contrast-to-tissue echo enhancement," ultrasound in Med. \& Biol., Vol. 24, no. 3, pp. 395-399, 1998.

[3] A.I. Eller," Subharmonic response of bubbles to underwater sound," J. Acoust. Soc. Am., Vol. 55, no. 4, pp. 371-373, 1974.

[4] C.K. Holland and R. E.Apfel, "An Improved Theory for the Prediction of Microcavitation Thresholds," IEEE Trans, Ultrason., Ferroelect., Freq. Contr., Vol. 36, No. 2, pp. 204-208, 1989.

[5] W.T. Shi and F. Forsberg," Ultrasonic Characterzation of the nonlinear properties of contrast microbubbles," ultrasound in Med. \& Biol., Vol. 26, no. 1, pp. 93-104, 2000.

E-mail: d88942007@ntu.edu.tw 